\title{
Towards the end of the Spanish paradox?
}

Dr. Juan Ruiz is Privat Docent and practices at the Endocrinology and Diabetology Unit of the University Hospital of Lausanne (Switzerland)

For many decades, we have known the association between cardiovascular disease and atherogenic lipid profile (low HDL-cholesterol, high LDL-cholesterol and high Lp (a) levels). The great majority of these studies have been performed on adult subjects. However, studies of autopsies made on young soldiers killed during the Korean War revealed the presence of aortic fatty streaks. Therefore, the atherosclerosis theory suggests that the development of the atherosclerotic plaque may occur in a very early stage of life. Recent studies confirm this assertion showing the presence of fatty streak and fibrous plaques among children and teenagers. Other studies clearly showed the association between teenagers having a high risk lipid profile and the development of coronary artery disease in young adults. These data suggest that an early screening of teenagers at risk could reduce the incidence of future cardiovascular events. Therefore, the AVENA study is important (see article in this issue, Ruiz et al). The main objective of this study was to provide references values for the serum lipids among Spanish teenagers. This type of study is precious because they are not numerous. The AVENA study is also essential because it concerns European teenagers, which will enable us to compare various regions from the South of Europe. The results of the AVENA study are very similar to the ones observed in the meta-analysis performed in Spain fifteen years before by Plaza et al. However, important differences are to be underlined. For the Spanish teenagers, the HDLcholesterol levels were slightly lower than the ones measured two decades before and the total cholesterol levels were slightly higher compared to the data from Greece. These data suggest that Spain is loosing its cardiovascular advantage, which was well-known as the Spanish paradox.

The process of acculturation (the modification of the culture of a group or an individual as a result of a contact with a different culture) is not only related to the migratory phenomena but it also is a result of the globalization. The Spanish life-style is changing as in most industrial countries. Nowadays, the consumption of fat among Spanish children and teenagers is increasing, while their vegetable and fruit intake is decreasing. They also reduce their physical activities. A raise of obesity and metabolic syndrome are the consequences expected for the next decades. These very fast life-style changes will have dramatic consequences on the incidence of cardiovascular disease. The red light is on: about ten percent of the US teenagers suffer from metabolic syndrome and two thirds present at least one metabolic abnormality. Biomarkers of increased risk of cardiovascular disease are already present in these young subjects. The AVENA study should be the first step for early prevention of cardiovascular disease. We hope that the future recommendations are going to promote a change of life-style rather than prescriptions of pills or "polypills". The success of such a strategy cannot take place without the creation of a real health promotion politic in European countries.

Juan Ruiz

\section{References}

Plaza I et al. (1990). Fuenlabrada study: lipid and lipoprotein levels in children and adolescents associated with ischemic cardiopathy prevalence among their relatives. Rev Esp Cardiol 43(4): 212-8.
Ruiz JR et al. (2006). Reference values for serum lipids and lipoprotein in Spanish adolescents: the AVENA Study. Soz Praventiv Med 51: 99-109.
Address for correspondence

PD Dr. med. Juan Ruiz CHUV - Centre Hospitalier Universitaire Vaudois

Rue du Bugnon 46

CH-1011 Lausanne

Soz Praventiv Med. 51 (2006) 79

0303-8408/06/020079-1

DOI 10.1007/s00038-005-0018-4

(c) Birkhäuser Verlag, Basel, 2006 